\title{
Robust Digital Image Reconstruction via the Discrete Fourier Slice Theorem
}

\author{
Shekhar S. Chandra, Member, IEEE, Nicolas Normand, Andrew Kingston, Jeanpierre Guédon, and Imants Svalbe
}

\begin{abstract}
The discrete Fourier slice theorem is an important tool for signal processing, especially in the context of the exact reconstruction of an image from its projected views. This paper presents a digital reconstruction algorithm to recover a two dimensional (2-D) image from sets of discrete one dimensional (1-D) projected views. The proposed algorithm has the same computational complexity as the 2-D fast Fourier transform and remains robust to the addition of significant levels of noise. A mapping of discrete projections is constructed to allow aperiodic projections to be converted to projections that assume periodic image boundary conditions. Each remapped projection forms a 1-D slice of the 2-D Discrete Fourier Transform (DFT) that requires no interpolation. The discrete projection angles are selected so that the set of remapped 1-D slices exactly tile the 2-D DFT space. This permits direct and mathematically exact reconstruction of the image via the inverse DFT. The reconstructions are artefact free, except for projection inconsistencies that arise from any additive and remapped noise. We also present methods to generate compact sets of rational projection angles that exactly tile the 2-D DFT space. The improvement in noise suppression that comes with the reconstruction of larger sized images needs to be balanced against the corresponding increase in computation time.
\end{abstract}

Index Terms-Discrete Fourier slice theorem, discrete Radon transform, discrete tomography, image reconstruction, Mojette Transform.

\section{INTRODUCTION}

$\mathbf{T}$ HE Discrete Fourier Transform (DFT) is an important tool for various signal processing problems ranging from filtering and convolution to inverse problems, where it is used as a mechanism to recover an object from its discrete projected "views" or projections [1].

The Discrete Fourier Slice Theorem (FST) property of the DFT, independently developed by Grigoryan and others [2]-[5], provides an exact partitioning of two dimensional (2-D) DFT space into a finite number of one dimensional (1-D) discrete

Manuscript received December 06, 2013; accepted March 17,2014. Date of publication March 24, 2014; date of current version April 02, 2014. The work of N. Normand was supported by Australian Research Council International Fellowship (ARC LX 0989907). The associate editor coordinating the review of this manuscript and approving it for publication was Prof. Kyoung Mu Lee.

S. S. Chandra is with the University of Queensland, Brisbane, St Lucia QLD 4072, Australia (e-mail: Shekhar.Chandra@uq.edu.au).

I. Svalbe is with Monash University, Victoria 3800, Australia (e-mail: Imants. Svalbe@monash.edu).

A. Kingston is with Australian National University, Canberra, ACT 0200 , Australia.

J. Guédon and N. Normand are with LUNAM Université, Université de Nantes, IRCCyN UMR CNRS 6597, Nantes, France.

Color versions of one or more of the figures in this paper are available online at http://ieeexplore.ieee.org.

Digital Object Identifier 10.1109/LSP.2014.2313341

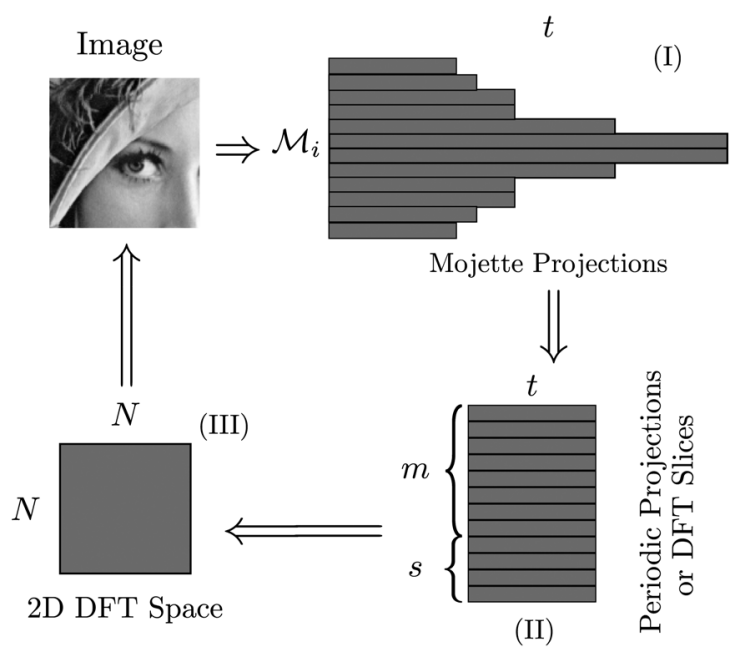

Fig. 1. A schematic of the main processes within the fast Mojette reconstruction. The Mojette projections (I), acquired using a new Mojette set definition, are converted to the periodic projections (II) via a new discrete projection mapping, and placed into Discrete Fourier Transform space (DFT) space (III) ready for inversion. Parts (I) \& (II) can be a logical step, i.e. the projections can be converted in-place.

"slices" of equal length. In this work, we present a method for recovering an image from a number of its discrete (rational angle, aperiodic) projected views using the slice theorem of the DFT that is both fast and robust in the presence of noise. The projected views are those of the discrete linogram also known as the Mojette Transform (MT) [6]. The proposed method allows these discrete projections to be exactly packed within the slices of the DFT, so that the inverse 2-D DFT is used to recover the image without any reconstruction artefacts apart from those formed from noise (see schematic in Fig. 1). An optimally compact rational angle set is also constructed for this reconstruction algorithm to improve response to noise and reduce its computational complexity.

The paper is structured as follows. A brief introduction to the discrete FST is made and the case for a digital reconstruction scheme for discrete rational angle projections made in the remaining parts of this section. Section II presents the proposed method and Section III discusses the results of this method.

\section{A. Discrete Fourier Slice Theorem}

The discrete FST, also known as the Finite Radon Transform, is a property of the DFT discovered and rediscovered a number of times over the last 150 years [7]-[10], [2]-[5], [11], [12]. The slices resulting from this theorem are the 1-D DFTs of periodic projections taken as sums along the lines

$$
\begin{aligned}
& y \equiv m x+t(\bmod N), \\
& x \equiv p s y+t(\bmod N),
\end{aligned}
$$


with slopes $m$ and $s$, intercepts $t$ and $x, y, m, s, t \in \mathbb{Z}$ for a square image of size $N=p^{n}$, where $p$ is prime and includes the powers of two [12]. The lines (1) \& (2) are equivalently formed by the vectors $[1, m]$ and $[p s, 1]$, i.e. $m$ pixels across and one pixel down or one pixel across and $p s$ pixels down. Rectangular $\left(N_{1} \times N_{2}\right)$ images have to be zero padded to the nearest square size.

The lines (1) and (2) are utilised for the set of slopes

$$
\begin{aligned}
\boldsymbol{m} & =\left\{m: m<N, m \in \mathbb{N}_{0}\right\}, \\
\boldsymbol{s} & =\left\{s: s<N / p, s \in \mathbb{N}_{0}\right\}
\end{aligned}
$$

and the set of translates

$$
\boldsymbol{t}=\left\{t: t<N, t \in \mathbb{N}_{0}\right\}
$$

where $\mathbb{N}_{0}$ represents whole numbers, i.e. the set of natural numbers including zero. To completely tile all elements of the entire space at least once, i.e. cover all possible coefficients in the $N \times N$ DFT space, a total of $N+N / p$ slices (and hence projections) are required [13]. For the simplest prime case $n=1$, $N+1$ projections are required and space is then tiled exactly [11]. For the case $n>1, N+N / p$ projections are required resulting in a certain amount of oversampling, which is easily and exactly corrected by dividing each coefficient $u$ for all $N+N / p$ slices by $\operatorname{gcd}(u, N)$ [13]. Orthogonal forms of the discrete FST also exist which do not require this sampling correction [14], [15]. The image can then be recovered from the projections by computing the 1-D DFT of these projections, placing these slices along the vectors $[-m, 1]$ and $[1,-p s]$ into 2-D DFT space directly (without interpolation) and computing the inverse 2-D DFT.

The discrete FST has been successfully applied to classical tomographic geometries, but no algorithm has resulted that is experimentally suitable and computationally practical [16]-[19]. Chandra [20] also showed that the projections can be mapped to the number theoretic transform, an integer only transform analogous to the DFT but with better performance for digital data. See Chandra et al. [21] for a recent detailed description of the discrete FST.

Unfortunately in most practical cases, only aperiodic discrete projections (generally from the MT [6]) exist in various signal and image processing applications ranging from psychovisual analysis [6], data integrity [22], [23], packet networks via an $n$-dimensional MT [24], lossless networking [25], image compression [26], scalable multimedia distribution [27], image coding [28] and watermarking [29], [30]. Recent efforts have also been made to apply the MT to real data with the aim of reducing the number of projections and thus reducing the dose to the subject or object being scanned [31], [32], [18], [19], [33]. Fig. 2 shows a simple example of a MT for a $4 \times 4$ image using three projections. See Guédon [34] for a detailed review of the MT.

A number of schemes have been proposed to recover images from these types of projections, including a conjugate gradient method [36] and a geometric graph approach [35]. The former is robust in the presence of noise but is not suitably convergent, i.e. an appropriate pre-conditioner is yet to be found, and the latter is very sensitive to noise. Chandra et al. [21] recently proposed an algorithm that recovers missing slices of the DFT applied

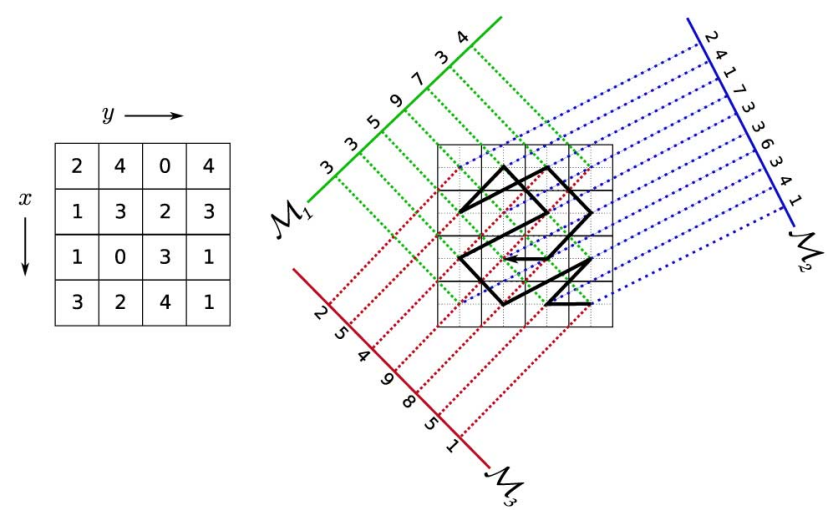

Fig. 2. An example of a Mojette Transform for a discrete image of size $4 \times 4$ using the three projections $[1,1],[1,-1]$ and $[1,-2]$. The bold lines within the right-hand grid shows a possible reconstruction path using a corner-based inversion method [35].

expressly for this purpose. However, their method requires an accurate estimate of the noise in order successfully handle artefacts created. In the next section, we present a fast algorithm for image reconstruction from noisy Mojette projected views.

\section{FAst Mojette ReCONSTRUCTION}

Given an $N^{\prime} \times N^{\prime}$ image of a digital object, the objective is to exactly tile an $N \times N$ DFT space, where $N=k N^{\prime}$ and $k \geq 1$, so as to efficiently reconstruct the image with the inverse DFT (iDFT). For the DFT space where $k>1$, redundancy provides the mechanism for suppressing the effects of noise within the projections.

Let the discrete projections be acquired digitally, i.e. assuming a Dirac pixel model, so that the pixel is summed to its corresponding bin if and only if the line passes through the centre of the pixel. The lines of the digital MT then form a set of non-periodic and parallel discrete lines

$$
\Gamma_{t, \theta_{p q}}=\left\{\begin{array}{lll}
t=q y-p x & \text { if } & q / p \geq 0 \\
t=p x-q y & \text { if } & q / p<0
\end{array},\right.
$$

where the projection is taken at angle $\theta_{p q}=\tan ^{-1}(q / p)$ (or the Farey vectors $[q, p]$ ) with $p, q, t \in \mathbb{Z}$ of an object having convex support. Convex support simply means that space does not have any local singularities along digital lines within the space.

Assuming that the size of the image is $N=2^{n}$, in order to utilise the Cooley-Tukey [37] algorithm for the DFT, let the total number of MT projections $M=N+N / 2$ and thus over-satisfy exact reconstruction requirements [38], [28]. Each Mojette projection is then mapped to a unique periodic projection or DFT slice to tile all of DFT space. Once the mapping is completed, the Mojette projections are converted to periodic projections ${ }^{1}$. A schematic of these processes are given in Fig. 1.

\section{A. Finite Projection Mapping}

The analytical mapping is as follows. For the case $n=1$ in $N=p^{n}$, the projection $\mathcal{M}_{\theta_{p q}}$ of the MT maps to the $m$ periodic projection as

$$
m \equiv p q^{-1}(\bmod N),
$$

\footnotetext{
${ }^{1}$ Implementations of this MT can be found as part of an open-source library (written by author SC) [39], including all algorithms discussed in this paper.
} 


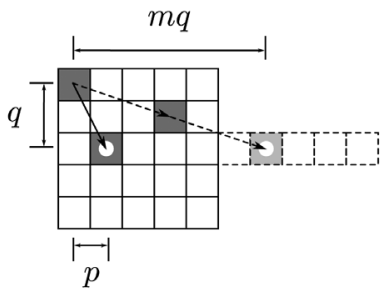

(a)

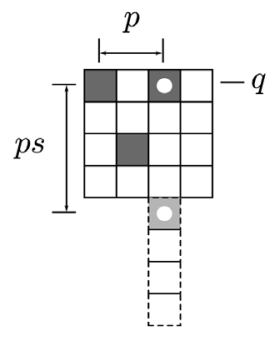

(b)
Fig. 3. A visual presentation of the mapping of Mojette Transform projections to the periodic projections of the DFT. Solving for $m$ in (a) and $s$ in (b) results in the mappings (7) and (8).

where $q^{-1}$ is the multiplicative inverse of $q$ (which can be computed easily via the Extended Euclidean algorithm). For the case $n>1$, the projection $\mathcal{M}_{\theta_{p q}}$ maps to the $m$ and $s$ projections as Eq. (7) when the $\operatorname{gcd}(p, N)>1$, and

$$
2 s \equiv p^{-1} q(\bmod N),
$$

when the $\operatorname{gcd}(q, N)>1$, respectively. These mappings cover all possible vectors/angles because the $\operatorname{gcd}(p, q)=1$ by definition of the angles. The mapping results from solving $m q \equiv$ $p(\bmod )$ and $2 s p \equiv q(\bmod )$ respectively. Examples of how each of these congruences are determined is shown in Fig. 3.

It is crucial to ensure that the Mojette set has a one-to-one correspondence to the periodic projection set, so that the DFT space is filled completely. The next section defines a new Mojette discrete angle set that is finite, fully tiling and spans the range $[0, \pi)$.

\section{B. Angle Set}

The Mojette projection set has to be constructed so as to fill DFT space completely with as little redundandacy as possible. The number of bins $B$ in a Mojette projection depends on the angle $\theta_{p q}$ as

$$
B=|p|(Q-1)+|q|(P-1)+1
$$

of a rectangular $P \times Q$ image. Minimising the $\ell_{1}$-norm of the Farey fractions/vectors as

$$
\ell_{1}=|p|+|q|
$$

is thus desirable, to keep the projections as compact as possible. The simplest way to achieve this is to generate angles through the vectors $[1, w]$ with $w \in \mathbb{Z}$ in all octants of the half plane. The $m$ or $s$ value is then checked with the periodic projection set in question for each vector $[1, w]$ using the mappings (7) and (8). An example of this set for an $8 \times 8$ image is given in Fig. 4(a). Note that this set is not the same as the periodic projection vectors $[1, m]$ and $[p s, 1]$, but shares their simplicity. These angles are a subset of the full set of all possible minimal $\ell_{1}$ vectors and is computationally inexpensive to generate. Hence, the set is well suited when adapting to a changing image geometry.

To generate the full minimal $\ell_{1}$ angle set, one computes

$q_{3}=\left\lfloor\frac{q_{1}+p_{1}+N}{q_{2}+p_{2}}\right\rfloor q_{2}-q_{1}, \quad p_{3}=\left\lfloor\frac{q_{1}+p_{1}+N}{q_{2}+p_{2}}\right\rfloor p_{2}-p_{1}$,

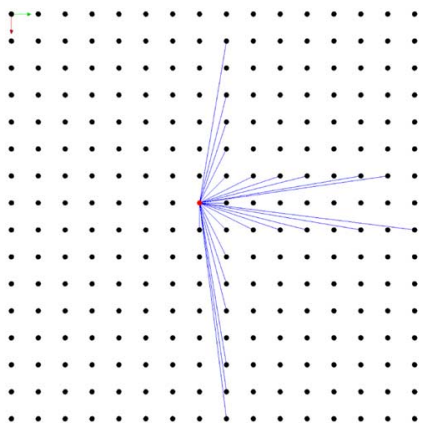

(a)

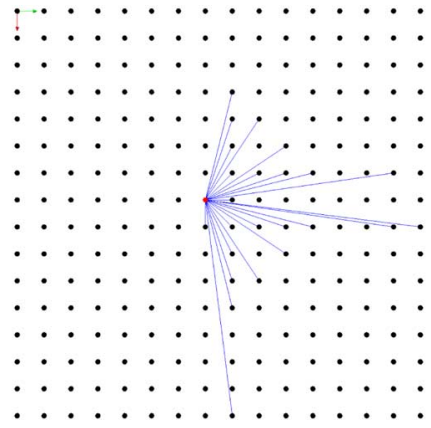

(b)
Fig. 4. (a) shows an example of a simple angle set, formed from the vectors $[1, a]$ with $a=0, \ldots, N-1$ in all octants in the half plane, used within the fast Mojette reconstruction for $8 \times 8$ array. Note that this set is not necessarily symmetric in all octants. (b) shows an example of a true $\ell_{1}$ minimised angle set for the same array. Both mappings tile or cover DFT space for the image exactly. The origin is marked as a red point

where $\lfloor\cdot\rfloor$ is the floor (round-down) operator, beginning the computation with $\left[q_{1}, p_{1}\right]=[1,0]$ and $\left[q_{2}, p_{2}\right]=[N, 1]$ until $\left[q_{3}, p_{3}\right]=[1,1]$. Eq. (11) has been derived from a standard technique to generate Farey fractions [40]. An example of this set for an $8 \times 8$ image is given in Fig. 4(b). Once generated, the set must be sorted by ascending $\ell_{1}$ and the first $N+\frac{N}{p}$ mappings chosen as the periodic angle set. This set requires the generation of a large number of fractions and sorting of these fractions, making the computation expensive compared to the simple set, but this set can be pre-computed for unchanging image geometries.

\section{Finite Conversion}

Once the projection set is known for a given image and DFT space, the Mojette projections need to be converted to periodic projections. This can be done by equating Eqs in (6), as well as Eqs (1) and (2), with the mappings (7) and (8). For a Mojette translate $t_{\mathcal{M}}$ and a periodic projection translate $t_{\mathcal{R}}$, one gets

$$
t_{\mathcal{R}}=\left\{\begin{array}{lll}
q^{-1} t_{\mathcal{M}}(\bmod N), & \text { if } & \operatorname{gcd}(p, N)>1 \\
p^{-1} t_{\mathcal{M}}(\bmod N), & \text { if } & \operatorname{gcd}(q, N)>1
\end{array} .\right.
$$

The conversion can be done prior to the inversion of the Mojette projections, or as the projections are being acquired. The latter is more desirable as the Mojette projections are effectively "compacted", since the periodic projections are generally shorter than the Mojette projections and all have the same size. The next section discusses the results of the proposed method in the presence of noise.

\section{RESULTS}

Numerical simulations of the proposed method were conducted of a $128 \times 128$ image of Lena on a Intel Core2Duo $(2.4 \mathrm{GHz})$ processor. Fig. 5 shows an example of a simulated reconstruction using fast Mojette reconstruction of the image with $3 \%$ Gaussian noise present in the Mojette projections within a $256 \times 256$ DFT space (i.e. the redundancy parameter of $k=2$ ). The reconstruction (Fig. 5(b)) shows that the result is stable to moderate levels of noise, having a Peak-Signal-to-Noise (PSNR) of approximately $35 \mathrm{~dB}$, which is suitable precision for lossy image and video encoding. The errors present on the reconstruction are shown in Fig. 5(c), which has a Root Mean 


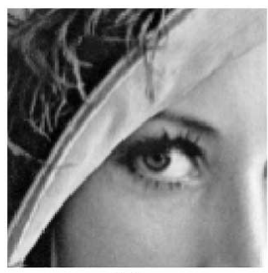

(a)
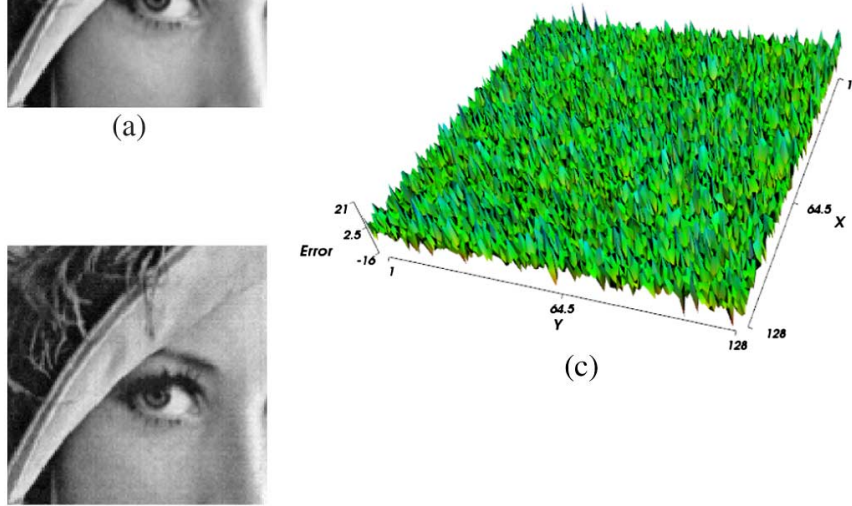

(c)

(b)

Fig. 5. A noise response numerical simulation of the fast Mojette reconstruction method using the simple angle set. (a) shows the original $128 \times 128$ image of Lena. (b) shows the reconstruction with $k=2$ and $3 \%$ Gaussian noise. (c) shows the actual errors between (a) and (b) as a surface plot. On average, the error is approximately 5 out of 256 grey-scales per pixel.

Squared Error (RMSE) of 4.8 grey-scales out of a possible 8-bit (256) grey-scales. Note that the noise is uniformly distributed and no image related artefacts are apparent.

Graph 6(a) shows the noise response of the two different angle sets as a function of the redundancy parameter $k$. The parameter values $k$ are defined as powers of two to ensure that the DFT space is also a power of two for the Cooley-Tukey algorithm [37]. Increasing the parameter $k$ spreads out the noise as predicted and exponentially decreases the error on the reconstruction. The simple angle set, which is a subset of the $\ell_{1}$ minimum set, has a better noise response because it possesses more bins on average than the full $\ell_{1}$ minimum set. The result is a more aggregation of bins due to the aperiodic-periodic mapping of Eq. (12).

Graph 6(b) shows the computation times of the MT and the fast Mojette reconstruction method in micro-seconds. The reconstruction times include the conversion of Mojette projections to slices (via Eq. (12) and 1-D DFTs) and the inverse 2-D $\mathrm{DFT}^{2}$. The reconstruction method, although an order of magnitude slower than the MT for very large images due to the number of DFTs involved, is suitably fast as the time for reconstructing a $4096 \times 4096$ image from 6144 projections is approximately 13 seconds. The time could be greatly reduced using Graphical Processing Units (GPUs). Considering both graphs, the optimal value for the parameter $k$ appears to be around $k=2$ or 4 , in order to balance noise response with the speed of computation.

Further work on the fast Mojette reconstruction method needs to be done in selecting optimal experimental geometries and conducting tomographic experiments with real data as well as comparing the effects of various detector noise types.

\section{CONCLUSION}

A well-conditioned algorithm for fast reconstruction of images from their discrete (rational angle) projections utilising the

${ }^{2}$ Computations of the DFT were done using the FFTW library [41].

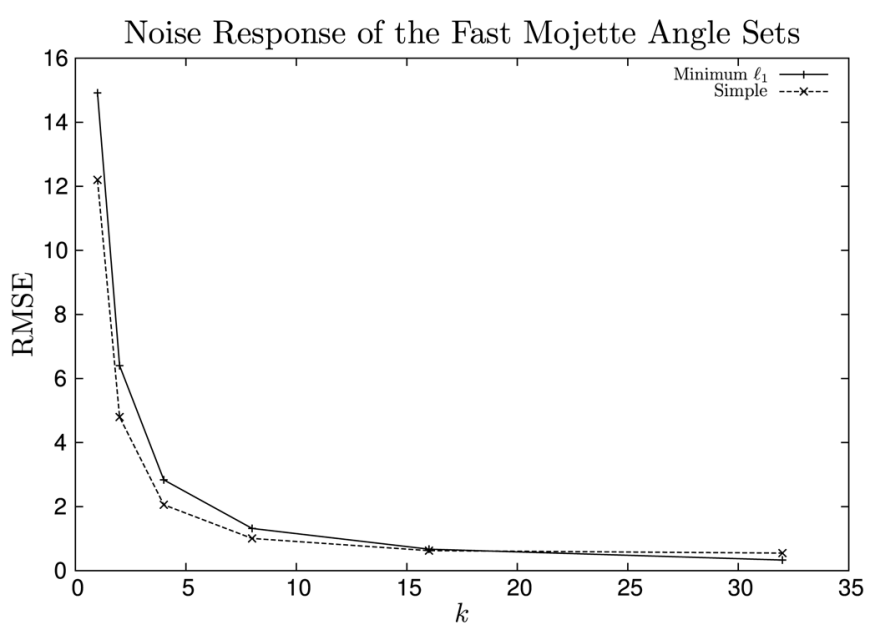

(a)

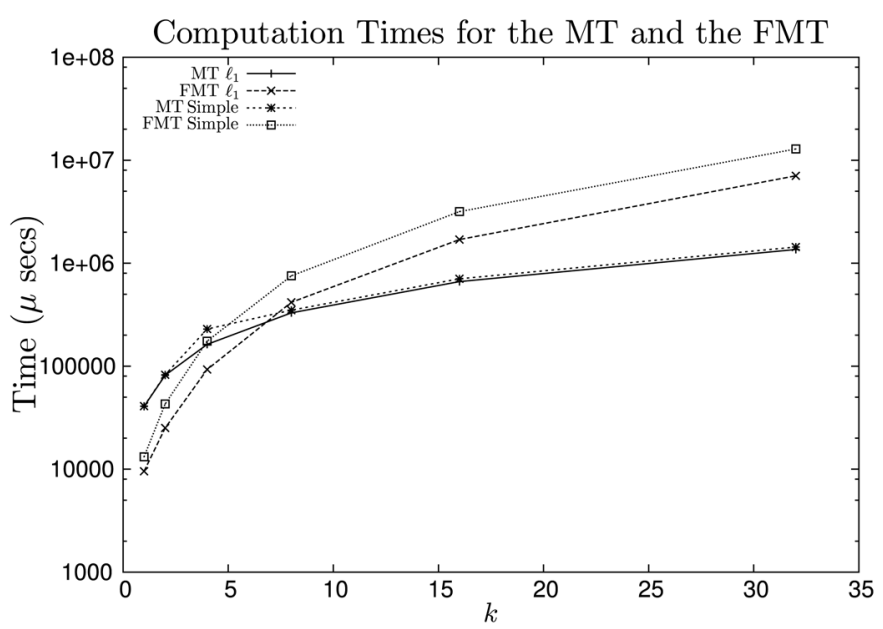

(b)

Fig. 6. A comparison of the angle sets for the fast Mojette reconstruction with a $128 \times 128$ image of Lena. (a) shows the Root Mean Squared Error (RMSE) with increasing redundancy $k$. (b) shows the computation times of the fast Mojette reconstruction method and the forward Mojette Transform (MT) on a log scale with the time in micro-seconds ( $\mu$ secs).

discrete FST was presented. A new analytical mapping of these projections was constructed that allowed them to be compacted directly and exactly into a Discrete Fourier space of desired size (see Eq. (12)). Once the projections are mapped, the (robust to noise) reconstruction can be obtained with a computational complexity $O\left(n \log _{2} n\right)$ with $n=N^{2}$ for an $N \times N$ space (see Fig. 5). Redundancy within this space was used to control inconsistencies in projection data, such as detector noise. The redundancy also allows the exploitation of $\ell_{1}$ or $\ell_{2}$-norm minimising (iterative) algorithms to values within the space to further reduce noise.

\section{ACKNOWLEDGMENT}

S. Chandra would like to thank the Faculty of Science and the School of Physics for a Ph.D. scholarship and a publications award. N. Normand would like to thank the Australian Research Council for his International Fellowship. 


\section{REFERENCES}

[1] G. T. Herman and A. Kuba, Discrete tomography: Foundations, Algorithms, and Applications. Boston, MA, USA: Birkhäuser, 1999

[2] A. Grigoryan, "New algorithms for calculating the discrete Fourier transforms," J. Vichislit. Matem. i Mat. Fiziki, vol. 25, no. 9, pp. $1407-1412,1986$.

[3] E. D. Bolker, "The finite Radon transform," Contemporary Math. (AMS), vol. 63, pp. 27-49, 1987.

[4] I. Gertner, "A new efficient algorithm to compute the two-dimensional discrete Fourier transform," IEEE Trans. Acoust., Speech, Signal Process., vol. 36, no. 7, pp. 1036-1050, 1988, 10.1109/29.1627.

[5] J. A. Fill, "The Radon transform on $\mathbb{Z}_{n}$," SIAM J. Discr. Math., vol. 2, no. 2, pp. 262-283, 1989, 10.1137/0402023.

[6] J.-P. Guédon, D. Barba, and N. Burger, "Psychovisual image coding via an exact discrete Radon transform," in Proc. SPIE , 1995, vol. 2501, pp. $562-572$.

[7] C. Jordan, Traité des substitutions et des équations algébriques. Paris, France: Gauthier-Villars, 1870.

[8] J. P. S. Kung, "The Radon transforms of a combinatorial geometry, I," J. Combin. Theory A, vol. 26, no. 2, pp. 97-102, 1979, 10.1016/00973165(79)90059-1.

[9] V. Labunets, "Superfast multidimensional Fourier Radon transforms and multidimensional convolutions," Statist. Meth. Signal Process., vol. IX, pp. 140-142, 1985.

[10] P. Diaconis and R. L. Graham, "The Radon transform on $\mathbb{Z}_{2}^{k}$," Pac. J. Math., vol. 118, pp. 323-345, 1985.

[11] F. Matúš and J. Flusser, "Image representation via a finite Radon transform," IEEE Trans. Patt. Anal. Mach. Intell., vol. 15, no. 10, pp. 996-1006, 1993, 10.1109/34.254058.

[12] T. Hsung, D. Lun, and W.-C. Siu, "The discrete periodic Radon transform," IEEE Trans. Signal Process., vol. 44, no. 10, pp. 2651-2657, 1996, 10.1109/78.539055.

[13] A. Kingston and I. Svalbe, "Generalised finite Radon transform for NxN images," Image Vis. Comput., vol. 25, no. 10, pp. 1620-1630, 2007, 10.1016/j.imavis.2006.03.002, Discrete Geometry for Computer Imagery 2005.

[14] D. Lun, T. Hsung, and T. Shen, "Orthogonal discrete periodic Radon transform. Part I: Theory and realization," Signal Process., vol. 83, no. 5, pp. 941-955, 2003, 10.1016/S0165-1684(02)00498-X.

[15] A. Kingston, "Orthogonal discrete Radon transform over $p^{n} \times p^{n}$ images," Signal Process., vol. 86, no. 8, pp. 2040-2050, 2006, 10.1016/j. sigpro.2005.09.024, Special Section: Advances in Signal Processingassisted Cross-layer Designs.

[16] P. M. Salzberg and R. Figueroa, Tomography on the 3D-torus and crystals G. T. Herman and A. Kuba, Eds., Discrete Tomography: Foundations, Algortihms and Applications Boston, MA, USA, Birkhäuser, 1999, ch. 19.

[17] I. Svalbe and D. van der Spek, "Reconstruction of tomographic images using analog projections and the digital Radon transform," Lin. Alg. Applicat., vol. 339, pp. 125-145, 2001, 10.1016/S00243795(01)00487-6.

[18] S. S. Chandra, I. Svalbe, and J.-P. Guédon, "An exact, non-iterative mojette inversion technique utilising ghosts," in Lecture Notes in Computer Science (LNCS). Berlin/Heidelberg, Germany: Springer, 2008, vol. 4992, pp. 401-412.

[19] H. Fayad, J. P. Guedon, I. Svalbe, Y. Bizais, and N. Normand, “Applying mojette discrete Radon transforms to classical tomographic data," Med. Imag., Phys. Med. Imag., vol. 6913, no. 1, p. 69132S, 2008, 10.1117/12.770478.

[20] S. S. Chandra and I. D. Svalbe, "Exact image representation via a number-theoretic Radon transform," IET Computer Vision, to be published.

[21] S. S. Chandra, I. Svalbe, N. Normand, A. Kingston, and J.-P. Guédon, "Recovering missing coefficients from discrete Fourier space using ghosts," IEEE Trans. Image Process., vol. 21, no. 10, pp. 4431-4441, 2012, 10.1109/TIP.2012.2206033.
[22] O. Philippé, J.-P. Guédon, and F. Terrien, "ATM source-channel image coding," Visual Communi. Image Process., vol. 3024, no. 1, pp. $1220-1230,1997,10.1117 / 12.263202$.

[23] F. Terrien, J.-P. V. Guédon, and O. Philippe, "Secure coding for medical sequence transmission over ATM network," Medical Imag. 1997: PACS Design Eval.: Eng. Clin. Issues, vol. 3035, no. 1, pp. 200-209, 1997, 10.1117/12.274572

[24] B. Parrein, P. Verbert, N. Normand, and J.-P. V. Guédon, "Scalable multiple descriptions on packets networks via the n-dimensional Mojette transform," Qual. Service over Next-Gen. Data Netw., vol. 4524 no. 1, pp. 243-252, 2001

[25] P. Verbert, J.-P. V. Guédon, and B. Parrein, "Distributed and compressed multimedia transmission using a discrete backprojection operator," Internet Multimedia Manag. Syst. III, vol. 4862, no. 1, pp. 315-325, 2002, 10.1117/12.473047.

[26] A. Kingston and F. Autrusseau, "Lossless image compression via predictive coding of discrete Radon projections," Signal Process.: Image Commun., vol. 23, no. 4, pp. 313-324, 2008, 10.1016/j.image.2008.03. 001 .

[27] J. Guédon, B. Parrein, and N. Normand, "Internet distributed image information system," Integr. Comput.-Aided Eng., vol. 8, no. 3, pp. 205-214, 2001.

[28] N. Normand, J.-P. Guédon, O. Philippe, and D. Barba, "Controlled redundancy for image coding and high-speed transmission," in Proc. SPIE , 1996, vol. 2727, pp. 1070-1081.

[29] F. Autrusseau, J. Guédon, and Y. Bizais, "Mojette cryptomarking scheme for medical images," in Proc. SPIE, 2003, vol. 5032, pp. 958-965, 10.1117/12.480296.

[30] M. Babel, B. Parrein, O. Deforges, N. Normand, J.-P. Guédon, and J. Ronsin, "Secured and progressive transmission of compressed images on the internet: Application to telemedicine," Internet Imag. VI, vol 5670 , no. 1, pp. 126-136, 2005.

[31] M. Servières, J.-P. Guédon, and N. Normand, "A discrete tomography approach to PET reconstruction," in Proc. 7th Int. Conf. Fully $3 D$ Reconstruction in Radiology and Nuclear Medicine, 2003.

[32] M. Servières, N. Normand, J.-P. Guédon, and Y. Bizais, "The Mojette transform: Discrete angles for tomography," in Proceedings of the Workshop on Discrete Tomography and its Applications. New York, NY, USA: Elsevier, 2006, pp. 587-606.

[33] B. Recur, H. D. Sarkissian, M. Servières, N. Normand, and J. Guédon, "Validation of mojette reconstructions from Radon acquisitions," in IEEE Int. Conf. Image Processing (ICIP), 2013.

[34] J. Guédon et al., The Mojette Transform: Theory and Applications, J. Guédon, Ed. : Wiley-ISTE, 2009.

[35] N. Normand, A. Kingston, and P. Évenou, "A geometry driven reconstruction algorithm for the mojette transform," in Lecture Notes in Computer Science (LNCS). Berlin / Heidelberg, Germany: Springer, 2006, vol. 4245, pp. 122-133.

[36] M. Servières, J. Idier, N. Normand, and J.-P. Guédon, "Conjugate gradient mojette reconstruction," in Proc. SPIE, 2005, vol. 5747, no. 1, pp. 2067-2074, 10.1117/12.593399.

[37] J. W. Cooley and J. W. Tukey, "An algorithm for the machine calculation of complex Fourier series," Math. Comput., vol. 19, no. 90, pp. 297-301, 1965, 10.2307/2003354.

[38] M. Katz, "Questions of uniqueness and resolution in reconstruction from projections," in Lecture Notes in Biomathematics. Berlin, Germany: Springer-Verlag, 1978.

[39] S. S. Chandra, "The finite transform library (FTL)," in $C / C++$ Library (Open Source under LGPL v3) 1.0. Australia: Monash . Press, 2009 [Online]. Available: http://finitetransform.sourceforge.net

[40] G. Hardy and E. Wright, An Introduction to the Theory of Numbers, 5th Ed. ed. Oxford, U.K.: Clarendon, 1979.

[41] M. Frigo and S. Johnson, "The design and implementation of FFTW3," Proc. IEEE, vol. 93, no. 2, pp. 216-231, 2005, 10.1109/JPROC.2004. 840301. 